\section{PRECISION ELECTRICAL MEASUREMENTS}

A SYMPOSIUM having as its general theme the techniques of precision electrical measurement was held at the National Physical Laboratory during November 17-20. Twenty-six papers were read and discussed, the authors being drawn from a particularly wide field, including not only some of the principal electrical laboratories in Britain but also members of the national laboratories of Australia, South Africa and the United States, and well-known workers from Germany, Holland and Sweden. In opening the proceedings, Dr. P. Dunsheath remarked that precision is evidently used as a relative term, for the errors recognized in, say, some of the magnetic and high-voltage techniques would fill those dealing with more manageable techniques with horror. Indeed, the subsequent discussion showed that mere statements of percentage accuracy are seldom of much interest; the important points are those which ensure that an experiment does in fact satisfy the conditions postulated in asserting that the quantity measured is such-and-such.

The symposium was divided into five sessions which dealt successively with subjects that form substantial items in the work of the Electricity Division of the National Physical Laboratory and that bear more or less directly on industry. The first session was devoted to the techniques applicable to electric fields and dielectrics; the second to magnetic fields and associated materials; the third dealt with electrotechnical measurements; the fourth with standard techniques for the high-voltage laboratory; and the last with the techniques of impulse-testing.

Perhaps the most outstanding feature of the techniques for the measurement of dielectric constant and power loss discussed in the first session was the range of frequency covered. Various aspects of the standard bridge and resonance techniques were introduced by Dr. L. Hartshorn and E. Rushton and J. V. L. Parry, of the National Physical Laboratory. Dr. M. Gevers, of the Philips Research Laboratories at Eindhoven, emphasized the versatility of standingwave methods for the microwave range, and it appears that these are superior to cavity resonator methods for materials of high loss, though the cavity has the advantage for low-loss materials. D. Woods, of the Ministry of Supply, showed that a bridge of the twin- $T$ type can be constructed to give high precision at frequencies up to $300 \mathrm{Me}$./s. At such frequencies, however, a massive block of brass used as the junction of two or more arms of the bridge is no longer an equipotential; it must be represented on the bridge diagram not as a mere junction point but as a network of small inductances. On paper, therefore, the bridge network opens out formidably, while the concrete reality shrinks to a closely interlocked group of brass blocks and cylinders the construction of which calls for mechanical work of very high precision. This indeed is a limitation of the technique, though its value for the particular purpose of calibrating standard coaxial components is obvious.

Considerable interest was also shown in techniques applicable to dielectric behaviour at extremely low frequencies. Dr. G. Mole and D. C. G. Smith, of the Electrical Research Association, described an a.c. bridge which is conveniently workable at frequeneies down to $0.05 \mathrm{c.} / \mathrm{s} . ;$ but the discussion showed that d.c. techniques are usually preferred for general work in this region, if only because, as C. G. Garton mentioned, time constants of the order of $50 \mathrm{hr}$. have been observed, and for periodic times of this order a.c. methods become quite impracticable.

Much of the discussion in the second session was concerned with various forms of permeameter used for determining the characteristics of industrial materials. C. E. Webb, of the National Physical Laboratory, dealt with standard methods for the delineation of magnetization curves. The discussion showed that the ballistic method is almost universally employed for the curves which serve as a basis for industrial design, and the discussion dealt largely with fine detail. Dr. M. McCaig, of the Permanent Magnet Association, stressed the difficulty of winding magnetic potentiometers with adequate uniformity and described a test that had revealed unsuspected errors in all such coils however carefully they had been wound. Prof. J. Greig and others mentioned the growing importance of methods for integrating and recording slow changes of flux and thus delineating individual cycles of magnetization, as a supplement to the ordinary curve for the steady cyclic condition that is determined point by point by the ballistic method; the two are clearly not necessarily the same and the differences are likely to be instructive. A paper by T. M. Palmer, of the Australian National Standards Laboratory, described a magnetometer in which a saturable core consisting of a short length of mumetal wire is used as a probe. The probe is small enough to serve as a null-detector for the measurement of $H$ in a permeameter and it therefore offers an interesting alternative to searchcoil methods; but its advantages have so far only been established for measurements of the earth's field and the like.

Methods for use at communication frequencies were described by A. C. Lynch, of the Post Office Engineering Research Station, and by P. H. Haas, of the U.S. National Bureau of Standards. Such methods depend on measurements of the inductance and equivalent resistance of toroidal cores provided with a suitable winding, the contribution of the winding alone being calculated or measured separately after removal of the core. The nature of the winding is an important factor; Mr. Haas places the core in a metal chamber which when closed by a cap forms a single short-circuited secondary turn; Mr. Lynch prefers a wire winding of three discrete turns; and Dr. C. M. van der Burgt, of the Philips Laboratories, showed an alternative scheme, by which a silver winding can be opened and closed for the insertion of the toroidal core, without introducing any uncertainty into the resistance of the winding, which is an extremely important factor.

A paper by R. L. Driscoll, of the U.S. National Bureau of Standards, described the determination at that laboratory of the magnetic moment of the proton (or more strictly its gyromagnetic ratio) by means of nuclear resonance. This brought microwave techniques and frequency measurement into the picture, and introduced the idea that the proton may conceivably serve as a magnetic standard accessible to everybody who can command the technique. The resonance frequency being determined only by the product of a constant characteristic of the proton and the field strength, it becomes possible to measure magnetic fields by observations of a 
frequency of resonance in water or any suitable material rich in protons used as a probe. At the National Bureau of Standards the field is actuatly measured in present-day units by weighing the force on straight conductors of accurately determined length suspended in it. The method is no doubt the best available for strong fields. For weaker fields it is probable that greater accuracy could be obtained by producing the field by passing a measured current through a primary standard inductor. The use of such an arrangement as a basis for magnetic measurements, and in particular for the calibration of search coils, was diseussed in a paper by G. H. Rayner, of the National Physical Laboratory.

The electrotechnical session began with the recognition that the accuracy of all measurements depends on the constancy of the quantities involved. Two aspects of the underlying problem were discussed, namely, the constancy of a.e. generator's and that of the alloys used for the construction of standard resistors. It became clear that electronically stabilized supplies are coming into general use in standards laboratories, and the system described by B. V. Hamon, of the Australian National Standards Laboratory, provided a good example; two 3-phase, $12 \mathrm{kVA}$. alternators have automatic control of both frequency and voltage to about 0.01 per cent at any setting in the working range. C. Petersen, of the U.S. National Bureau of Standards, reviewed the work on resistors, and great interest was shown in the subject. It became evident that an alloy of exceptionally high resistivity and adequate stability has been sought in vain by many, that manganin still holds its place for stability, with constantan as an alternative that is almost indispensable for a.c. work, and that modified nickelchromium alloys show promise of higher resistivity with stability. The remaining papers centred around the problem of the d.c. to a.c. transfer standard. In theory no standard is necessary; instantaneous values are measurable by direct reference to d.c. standards, but practice is mainly concermed with steady-state values, and the accuracy required is much greater than can be obtained for instantaneous values. Hence the importance of the stabilized supply mentioned earlier, and the transfer standard which indicates with precision either d.c. or stationary root mean square values. Examples of the three types now in use were discussed: a dynamometer wattmeter constructed by Dr. O. Brune and J. W. Whittaker at the National Physical Laboratory, South Africa; thermal instruments used as ammeters and voltmeters at the U.S. National Bureau of Standards described in a paper by F. L. Hermach; and the electrostatic wattmeter used at the National Physical Laboratory and described by Dr. A. H. M. Arnold with particular reference to work at audiofrequencies.

On proceeding to higher voltages, the same considerations apply with the additional complications that clearances must be made larger to avoid breakdown so that the equipment tends to become excessively bulky, resistors of extremely high value become necessary to avoid excessive power dissipation and transients as well as steady-state values are often required. The compressed gas capacitor, discussed by Dr. A. Keller, of Hartmann and Braun A.G., Germany, well illustrates one answer to the problem of keeping down the bulk of the equipment. Single units containing carbon dioxide at 14 atmospheres pressure have now been made to withstand an operating voltage of $900 \mathrm{kV}$. (r.m.s.), with a capacitance of $65 \mathrm{pF}$. They serve as standards for the measurement of dielectric loss at high voltage. G. W. Bowdler, of the National Physical Laboratory, discussed methods for the measurement of high voltages in steady-state conditions, especially the construction of resistors of high value, and potential dividers, and the use of the attracted disk electrostatic voltmeter, which with compressed gas insulation has been used up to $600 \mathrm{kV}$. (peak). P. R. Howard, of the National Physical Laboratory, dealt with the measurement of transients. It is, of course, the cathode-ray oscillograph that has made such measurements possible, but accuracy depends largely on the proper control and assessment of stray capacitance and inductance in the auxiliary equipment of voltage divider and cables between the instrument and the point of the system under investigation. F. S. Edwards, of the MetropolitanVickers Electrical Co., Ltd., discussed the sphere-gap as a peak voltmeter. Most speakers saw something incongruous in the basing of a standard on anything so erratic and violent as an electrie spark; but the fact that standard calibrations for the sphere-gap have been issued in Britain, the United States and Germany is evidence of its practical importance for the measurement of very high voltages and of the need for a knowledge of the ways of minimizing the errors and estimating their magnitude.

The final session was devoted to a discussion of impulse tests on high-voltage equipment. At first sight the problems are somewhat remote from precision measurement. The objective is a proof of the ability of a transformer, cable or other insulated system to withstand surges of voltage such as are liable to arise in practice from lightning strokes or switching operations. Surges of suitable severity must be generated, applied at specified points, and the currents or voltages at key points must then be examined for any departure from normal behaviour such as could arise from partial failure. The whole problem is of such intricacy, including as it does the recognition and interpretation of special features in transients in complicated systems of inductance, capacitance, etc., that the techniques are far less standardized than are those of a measurement. However, the discussion showed that the techniques have much in common with those of measurement. The distribution of transients within a complex network, for example, is determined by factors exactly similar to those in a high-frequency bridge. The problem of the common junction point in $\mathrm{Mr}$. Woods's bridge discussed in the first session has its counterpart in the common 'earth' of an impulsetesting system, and the proper location of earth capacitance and residual inductance provides the solution in both cases. The details, however, defy summary treatment. W. G. Standring, of the National Physical Laboratory, presented a general survey of the problem; N. Hylten-Cavallius, of A.S.E.A., Sweden, an account of the design and construction of surge generators ; G. H. Hickling, of C. A. Parsons and Co., Ltd., J. Neuve-Eglise, of Electricité de France, and P. G. Provoost, of K.E.M.A. Laboratories, Holland, each recorded experience of the detection of faults in transformers; and B. Salvage, of W. T. Henley's Telegraph Works Co., Ltd., discussed the corresponding problem for cables and their accessories.

The complete proceedings will be published as soon as practicable and will be obtainable from H.M. Stationery Office.
L. Hartshorn 